\title{
Statistical Properties of the Dissolution Test of USP
}

\author{
Carlos D. Saccone ${ }^{1,3}$, Julio Tessore ${ }^{1}$, Silvino A. Olivera ${ }^{2}$, \\ and Nora S. Meneces ${ }^{1}$
}

email:csaccone@fing.edu.uy

\begin{abstract}
The Monte Carlo simulation method is used to study statistical properties of the USP dissolution test. Some interesting aspects of immediate release dissolution are presented, including:

a. A unified operating curve that allows estimation of the probability of acceptance (Pa) of a lot as a function of its statistical parameters.

b. Verification that the statistical behavior is only slightly affected by the underlying distribution of the individual amounts dissolved.

c. The average number of samples required to reach a decision is presented as a function of parameters of the lot.

d. The relative influence of the three stages of the test in the probability of acceptance.
\end{abstract}

\section{Introduction}

he dissolution test as defined in the United States Phar-

macopoeia (1) is used in judging the quality of pharma-

ceutical products. Dissolution testing is a method for evaluating physiological availability that depends upon having the drug in a dissolved state. The USP Dissolution testing involves three stages and the acceptance criteria are defined for each stage as a function of a quantity $Q$, a percentage of the label value that is established for each drug product in its monograph. Acceptance criteria are shown in Table 1.

These acceptance criteria are complex and the behavior of the test for samples of varying quality levels is not easily predictable from the knowledge of its drug properties. Pharmaceutical manufacturers are interested in many of

Table 1. USP Acceptance Criteria

\begin{tabular}{|lcl|}
\hline Stage & $\begin{array}{c}\text { Number } \\
\text { units }\end{array}$ & \multicolumn{1}{c|}{ Acceptance Criteria } \\
\hline S1 & 6 & Each unit is not less than $\mathrm{Q}^{*}+5 \%$ \\
\hline S2 & 6 & $\begin{array}{l}\text { Average of the 12 (S1+S2) units is } \geq \\
\text { Q and no uni is less than Q-15\% }\end{array}$ \\
\hline S3 & 12 & $\begin{array}{l}\text { Average of 24 (S1+S2+S3) units is } \geq \\
\text { Q and not more than 2 units are } \\
\text { less than Q-15\% and no unit is less } \\
\text { than Q-25\% }\end{array}$ \\
\hline $\begin{array}{l}\text { *Q is the amount of dissolved active ingredient specified in the individ- } \\
\text { ual monograph, expressed as a percentage of the labeled content. }\end{array}$ \\
\hline
\end{tabular}

the statistical properties of the dissolution test. The following aspects of immediate release dissolution were studied:

- Probability of acceptance of the dissolution test $(\mathrm{Pa}), \mathrm{e}$. g. probability of passing the test, as a function of the dissolution population parameters (mean and standard deviation expressed as a percentage of label content),

- Influence of the shape of the population distribution on the probability of acceptance.

- Average sample number needed for reaching a decision when the test is applied.

- Contribution of each stage of the test to the probability of acceptance.

\section{Methodology}

The Monte Carlo simulation method was used to study statistical properties of the dissolution test. Amounts dissolved, expressed as a percentage of the label value of each unit (tablet, capsules, etc.), were obtained through the use of Visual Basic-Excel statistical routines. In Figure 8, a flowchart (similar to the flowchart presented by PHEATT (2) of the simulation is provided. More than 100 million dissolution values were generated in order to assure uncertainty values of less than 0.01 in the probability of acceptance.

Probability of acceptance, $(\mathrm{Pa})$, and average sample number (ASN) were studied in the range of conditions of interest for the objectives of this study, as shown in Table 2.

\section{Results and Discussion Operating Characteristic Curves}

The operating characteristic curves of the dissolution test are defined in this paper as Probability of acceptance

\footnotetext{
${ }^{1}$ University of the Republic of Uruguay, Faculty of Engineering, Industrial Production Department, Montevideo, Uruguay

${ }^{2}$ University of the Republic of Uruguay, Faculty of Chemistry, Estrella Campos Department, Montevideo, Uruguay
}

${ }^{3}$ Corresponding author, Facultad de Ingeniería, Instituto de Ingeniería
Mecánica y Producción Industrial, Julio Herrera y Reissig 565, Montevideo,
Uruguay

Dissolution Technologies | AUGUST 2004 


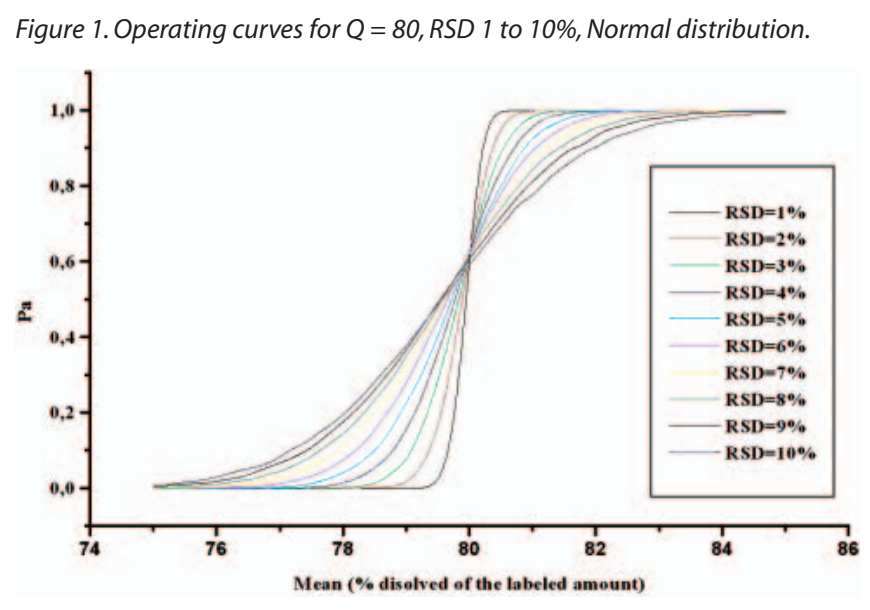

Figure 2. Plot of Probability of acceptance of dissolution test as a function of (Mean-Q)/Std. Deviation. Unified curve made by using all the values obtained for $Q=75$ and $Q=80$ and $R S D 1$ to $10 \%$.

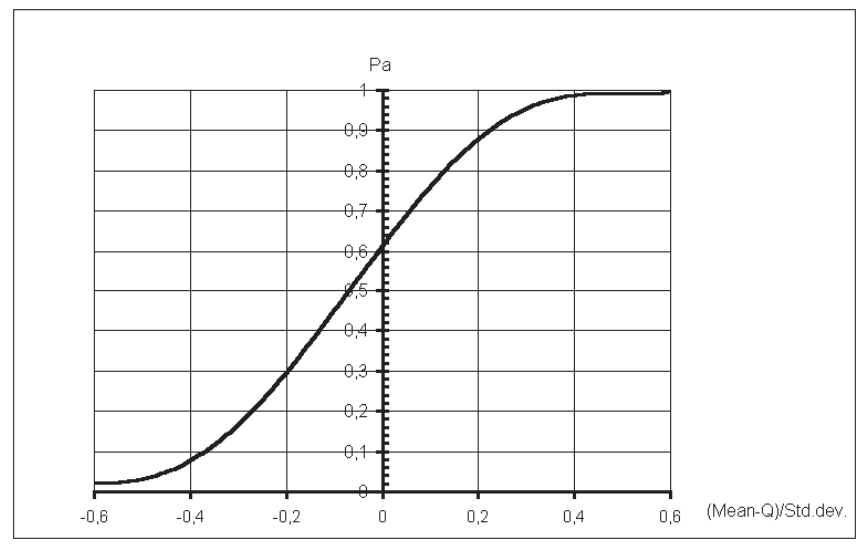

Figure 3. Plot of the differences between Probabilities of acceptance in dissolution test (Normal minus lognormal), assuming Normal and Lognormal distributions as a function of Mean, $Q=75$ and RSD 1 to $10 \%$. Solid dots were obtained for RSD between 1 and $8 \%$, empty dots were obtained for RSD 9 and $10 \%$.

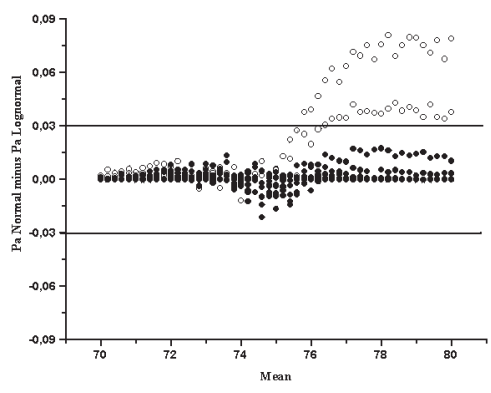

(Pa) vs. mean of dissolution values expressed as a percentage of the label content. They have a characteristic $\mathrm{S}$ shape, and are shown in Figure 1.

All the curves, in the range of RSD studied, intercept at a mean value equal to $Q$. This means that at this point, the Probability of acceptance does not depend on RSD and its value is $\mathrm{Pa}=0.62$. For $\mathrm{Q}=75$ a similar behavior is obtained in the shape of the curves and in the intercept value $(\mathrm{Pa}=0.62$, mean $=\mathrm{Q})$.
Table 2. Operation Conditions for studying probability of acceptance $(\mathrm{Pa})$ and the average sample number (ASN)

\begin{tabular}{|l|c|c|c|c|}
\hline \multirow{2}{*}{} & \multicolumn{2}{|c|}{$\mathrm{Q}=75$} & \multicolumn{2}{c|}{$\mathrm{Q}=80$} \\
\cline { 2 - 5 } & $\mathrm{Pa}$ & $\mathrm{ASN}$ & $\mathrm{Pa}$ & $\mathrm{ASN}$ \\
\hline Mean (\%) & $70-90$ & $50-90$ & $75-95$ & $55-95$ \\
\hline RSD (\%) & $1-10$ & $1-15$ & $1-10$ & $1-15$ \\
\hline Distributions & $\mathrm{N}, \mathrm{LN}^{* *}$ & $\mathrm{~N}, \mathrm{LN}$ & $\mathrm{N}, \mathrm{LN}$ & $\mathrm{N}, \mathrm{LN}$ \\
\hline $\begin{array}{l}\text { Uncertainty } \\
\text { in Pa }\end{array}$ & $\begin{array}{c}\text { Less than } \\
0.01\end{array}$ & & $\begin{array}{c}\text { Less than } \\
0.01\end{array}$ & \\
\hline
\end{tabular}

*RSD: Relative standard deviation

${ }^{* *} \mathrm{~N}, \mathrm{LN}$ : Normal and lognormal distribution.

\section{Unified Characteristic Curve}

As Murphy and Sampson (3) suggested, the curves may be unified representing $\mathrm{Pa}$ as a function of a parameter that eliminates the influence of $Q$ and RSD. The results obtained for Pa as a function of (Mean=Q)/Standard deviation are shown in Figure 2 . The curve was made by using all the values obtained for $\mathrm{Q}=75$ and $\mathrm{Q}=80$ and RSD 1 to $10 \%$. This curve thus built allows us to easily estimate $\mathrm{Pa}$ as a function of dissolution parameters (mean, standard deviation). The thickness of the curve indicates the maximum observed variations in the Pa obtained in the simulations performed. These variations observed represent less than approximately \pm 0.02 in the Pa in all the ranges studied.

It can be observed that when the mean value is Q-0.6 standard deviation and less, the Pa is insignificant, and when the mean value is $Q+0.6$ standard deviation and more, the $\mathrm{Pa}$ is almost 1.

\section{Robustness}

There is no agreement (2) about the shape of the distribution of the dissolved amounts and therefore it was considered important to study how the curves shown above depend on the distribution assumed. Particularly, for normal and lognormal distributions the different Pa have been evaluated. These distributions were chosen for the following reasons:

- Normal distribution was considered a good model of the distribution since the amount dissolved by each unit is a function of a large number of variables.

- Lognormal distribution seems suitable to simulate a physical limit to the amount dissolved due to the amount of drug product in the pharmaceutical dosage form. If the underlying distribution of amount dissolved is lognormal, a large slope is observed towards the right (where the physical limit exists) and a low slope towards the left.

The observed influence on probability of acceptance is shown in Figure 3, and it can be seen that there are not rele- 
vant differences between normal and lognormal distributions. The probability of passing the test when the data were normally distributed differs less than 0.03 from that of lognormal distribution for population RSD values of $8 \%$ or less.

As expected, in the range in which the rejections are due to the non-compliance of the requirements on the average value (stages 2 and 3), the central limit theorem assures insensitivity to the distribution shape. When non-compliances are due to individual values (clauses Q-25\% and Q$15 \%)$, the probability of acceptance is more dependent on the assumed distribution of amount dissolved. This occurs for larger RSDs, 9 and $10 \%$.

\section{Average Sample Number}

The average sample number of units required to arrive at a decision about the test was studied. This number (ASN) is a function of the mean and standard deviation.

Ideally, if it is considered that the variability is zero $(\mathrm{RSD}=0$ ), when the dissolution values increase (Figure 4) it should be expected that:

a. Rejection of tests in the first stage if dissolution is less than Q-15\% (tested units $=6$ )

b. Three stages are required to reach a decision with dissolution values less than Q and more than Q-15\% (tested units $=24$ )

c. Acceptance in the second stage for dissolution values that are more than $\mathrm{Q}$ and less than $\mathrm{Q}+5 \%$ (tested units $=12$ )

d. Acceptance in the first stage for dissolution values that are more than $Q+5 \%$ (tested units $=6$ )

The curves obtained with variability different to zero, (RSD 1 to 15\%) present the four steps described, but they separate from the ideal curve and differences are larger as the RSD increases (Figure 5).

Additionally, it must be said that the point with a mean $=\mathrm{Q}$ shows a behavior that is practically independent of RSD in the ranges studied: it requires 18 samples as an average. Also the behavior is almost independent of RSD when the mean is equal to $\mathrm{Q}+5 \%$ or $\mathrm{Q}-15 \%$ when $\mathrm{RSD}$ is less than $8 \%$.

\section{Contribution of each stage to the acceptance of the test}

In order to understand the operation of the dissolution test, contributions to the total probability of acceptance were studied for each stage of the test.

The following results were obtained:

- The first stage begins to contribute to the Pa when the mean is more than $\mathrm{Q}+5 \%$. As the mean increases and it approaches the label value this contribution becomes larger than those of other stages. See Figures 6 and 7.

- The third stage only contributes to the Pa when the mean is close to Q. For RSD 1 to $10 \%$ and when standard deviation is eight or less, the maximum contribution found for the third stage to the Pa was about 20\%.
Figure 4. Ideal curve. Average Sample Number as a function of Mean, $Q=75$ and $R S D=0 \%$.

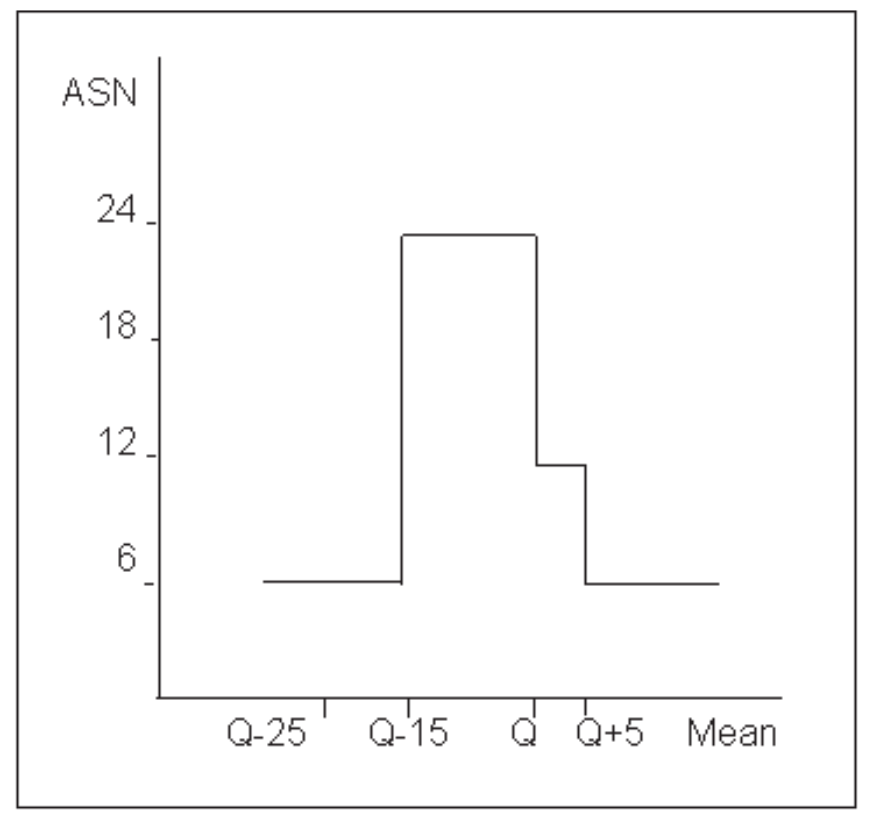

Figure 5. Plot of Average Sample Number as a function of Mean, $Q=75$ and RSD 1 to $15 \%$.

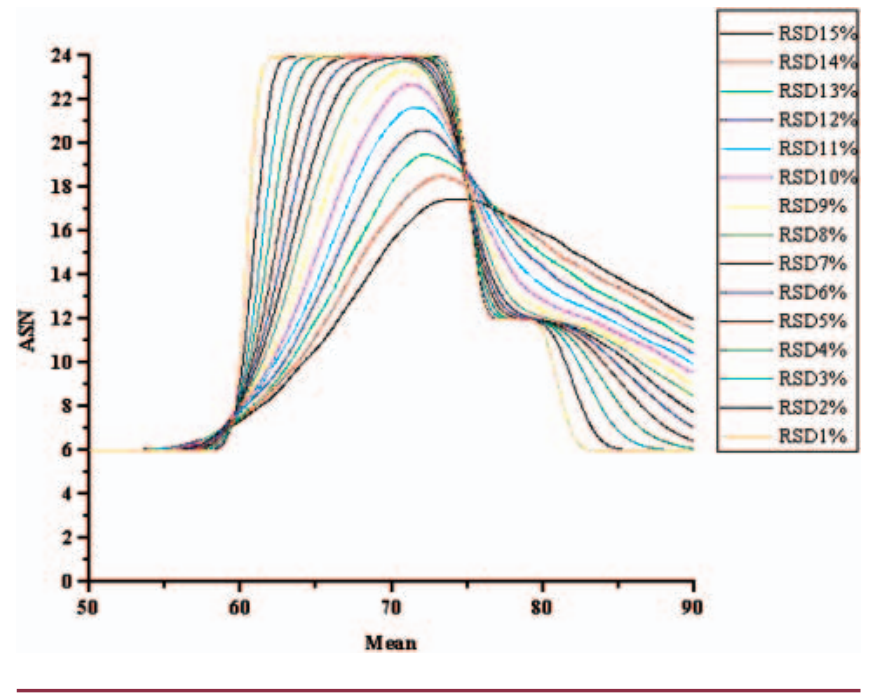

- In all the other situations, the acceptance decisions are produced in the second stage.

For $\mathrm{Q}=80$ a similar behavior to the above explained for $\mathrm{Q}=75$ is obtained.

\section{Conclusions}

a. A unified operating curve is presented that allows estimating the probability of acceptance ( $\mathrm{Pa}$ ) of the dissolution test as a function of the dissolution parameters that characterize it. This curve can be used to evaluate the probability of passing the test by the authority and, therefore, the risks of releasing lots of varying quality levels and its possible consequences. 
Figure 6. Plot of probability of acceptance of the dissolution test at Stage 1, Stage 2 and Stage 3 as a function of Mean, $Q=75$ and RSD $=5 \%$.

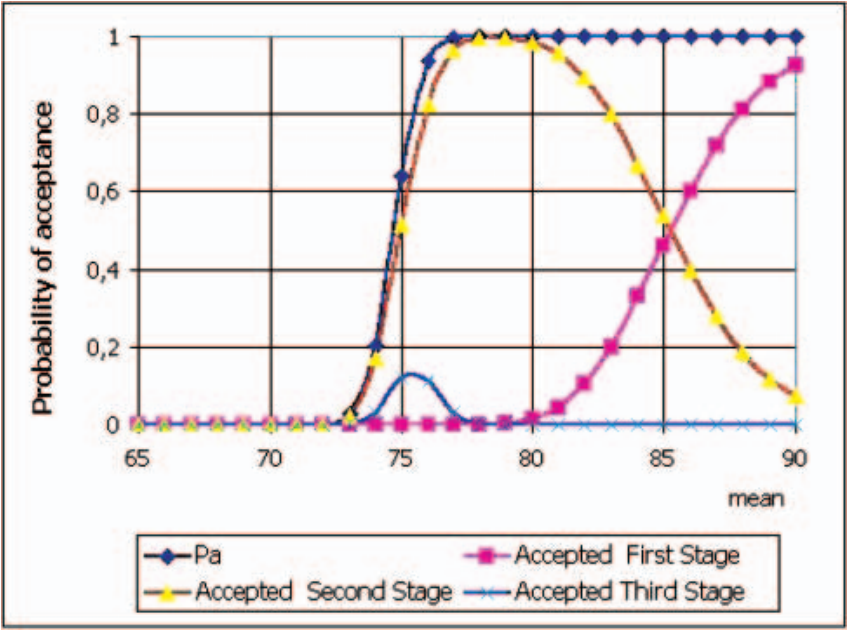

Figure 7. Plot of probability of acceptance of the dissolution test at Stage 1, Stage 2 and Stage 3 as a function of Mean, $Q=75$ and $R S D=10 \%$.

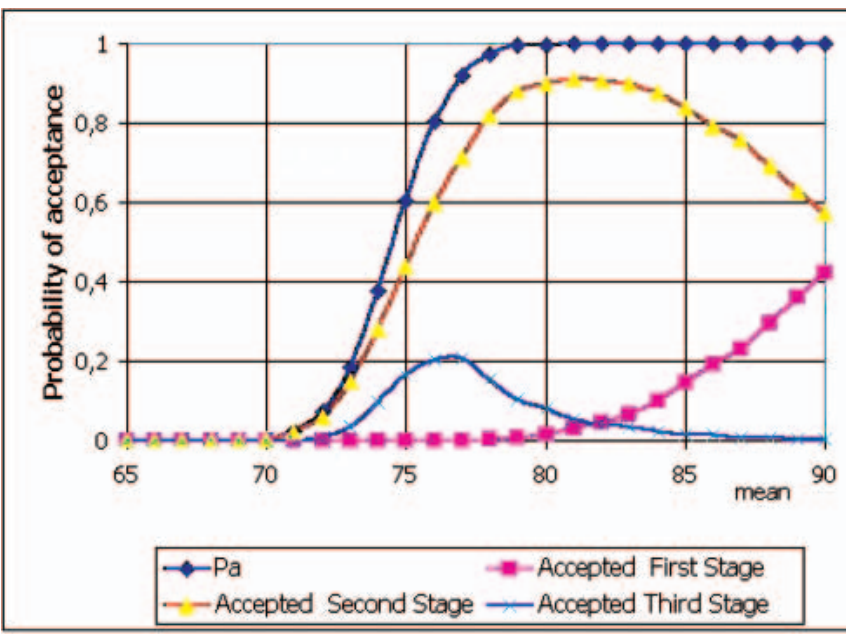

b. For means less than Q-0.6 6 , the probability of acceptance is practically zero. A mean value larger than $\mathrm{Q}+$ $0.6 \sigma$ assures the acceptance of tests. This value $(Q+0.6 \sigma)$ could be the release criteria used by the manufacturer, to minimize risk of rejection by the authority.

c. The statistical behavior does not depend on the shape of the distribution of the amounts dissolved, at least for standard deviation less than 7, considered customary by Hofer and Gray (4) in the dissolution test.

d. The number of tests required to reach a test decision depends on the population's dissolution parameters. As usual with double or multiple sampling plans, with very bad or very good lots, the decision of acceptance or rejection of the test is reached quickly and the number of units tested is minimum (6, only first stage).

e. Although the test involves three stages, the behavior is dominated by the first and second stage.
Figure 8. Flowchart of dissolution test simulation

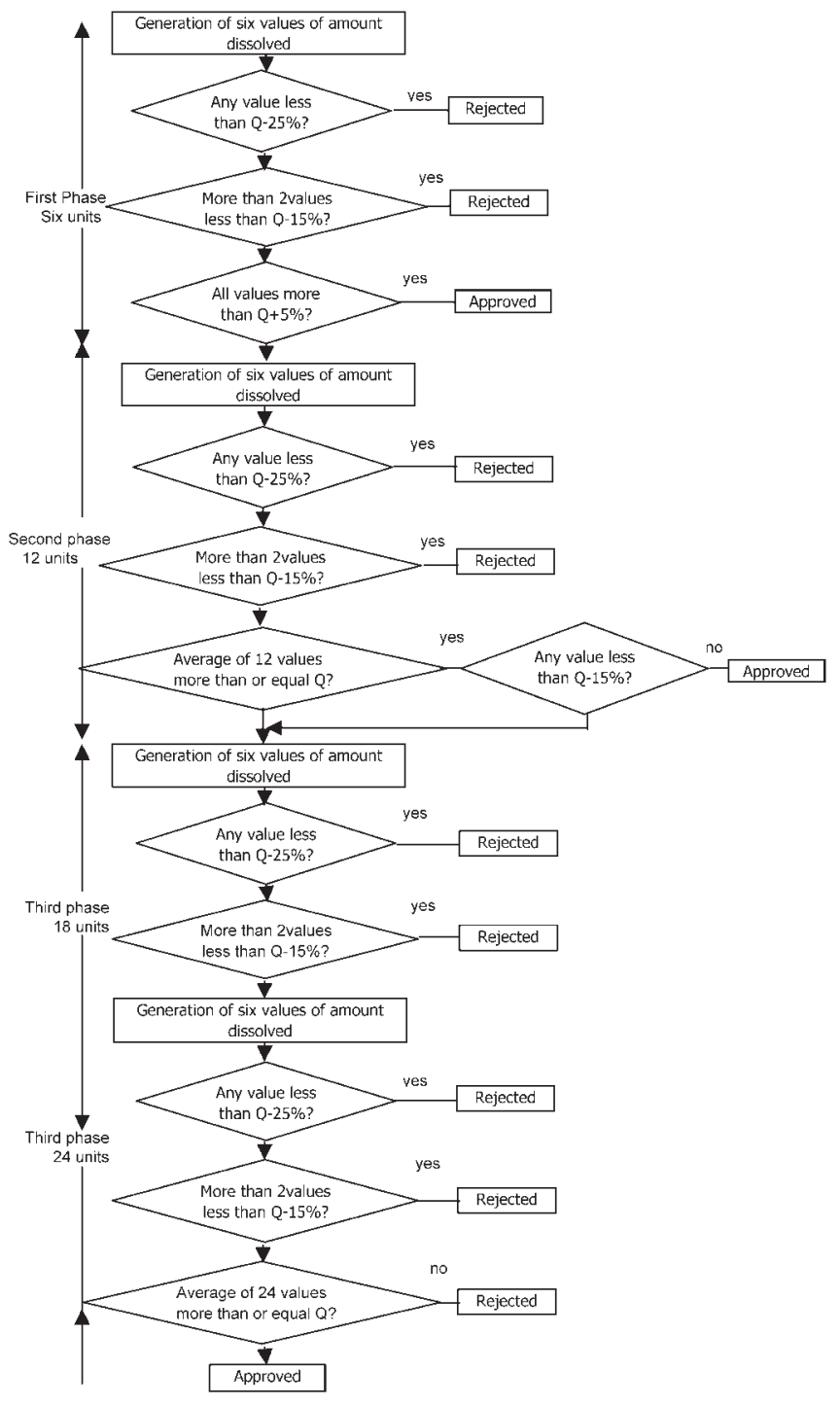

References

1. The United Stated Pharmacopoeia XXVI, The U. S. Pharmacopeial Convention, Inc., Board of Trustees, Webcom Limited, Toronto, Ontario, 2155-2156, 2003.

2. Pheatt, Charles B., "Evaluation of U.S. Pharmacopeia Sampling Plans for Dissolution", Journal of Quality Technology, 12 (3, July), 158-164, 1980.

3. Buncher, Ralph C., Tsay, Jia-Yeong, Statistics in the Pharmaceutical Industry, Statistics:Textbooks and Monographs, Marcel Dekker, Inc., 140, 402-406, 1994.

4. Hofer, Jeffrey D., Gray Vivian A, “Examination of selection of Immediate Release Dissolution Acceptance Criteria," Dissolution Technologies, 10 (1, February), 16-20, 2003. 